\title{
Improved Ocular Tissue Models and Eye-On-A-Chip Technologies Will Facilitate Ophthalmic Drug Development
}

\author{
Charles B. Wright, ${ }^{1}$ Steven M. Becker, ${ }^{1}$ Lucie A. Low, ${ }^{2}$ Danilo A. Tagle, ${ }^{2}$ and Paul A. Sieving ${ }^{1}$
}

\begin{abstract}
In this study, we describe efforts by the National Eye Institute (NEI) and National Center for Advancing Translational Science (NCATS) to catalyze advances in 3-dimensional (3-D) ocular organoid and microphysiological systems (MPS). We reviewed the recent literature regarding ocular organoids and tissue chips. Animal models, 2-dimensional cell culture models, and postmortem human tissue samples provide the vision research community with insights critical to understanding pathophysiology and therapeutic development. The advent of induced pluripotent stem cell technologies provide researchers with enticing new approaches and tools that augment study in more traditional models to provide the scientific community with insights that have previously been impossible to obtain. Efforts by the National Institutes of Health (NIH) have already accelerated the pace of scientific discovery, and recent advances in ocular organoid and MPS modeling approaches have opened new avenues of investigation. In addition to more closely recapitulating the morphologies and physiological responses of in vivo human tissue, key breakthroughs have been made in the past year to resolve long-standing scientific questions regarding tissue development, molecular signaling, and pathophysiological mechanisms that promise to provide advances critical to therapeutic development and patient care. 3-D tissue culture modeling and MPS offer platforms for future high-throughput testing of therapeutic candidates and studies of gene interactions to improve models of complex genetic diseases with no well-defined etiology, such as age-related macular degeneration and Fuchs' dystrophy.
\end{abstract}

Keywords: organoid, tissue chip, microphysiological systems

\section{Introduction}

$\mathbf{T}$ HE MISSION OF the National Eye Institute (NEI) at the U.S. National Institutes of Health (NIH) is to support research that helps prevent and treat eye diseases and other disorders of vision. NEI-supported research has advanced our knowledge of how visual system functions in both health and disease. Typically the tools employed by researchers studying development or disease have included animal models, fetal tissue, immortalized cell lines, or explants from cadaveric eyes. Recently the use of human stem cell-derived ocular tissue has enabled investigators to probe development and disease in new ways. Organoid technologies and microphysiological systems (MPS) enable scientific questions to be addressed that have been difficult, if not impossible, to study in vivo. Despite being a relatively new approach to modeling development and disease, advances in ocular organoids for the past year provide a preview of the kinds of scientific insights the vision research community can expect from these improved ocular tissue models.

\section{Recent Advances}

Significant advances have recently been made in both the culture conditions and experimental methodologies for generating and studying retina and cornea organoids. The number of publications citing retina organoids has grown significantly in the past 5 years. (Fig. 1). The NEI has sought to capitalize on this emerging technology and launched its 3-dimensional (3-D) Retina Organoid Ideation Challenge in 2017 with a follow-on prize competition referred to as the 3-D Retina Organoid Challenge 2020 that launched in early 2018 (3-D ROC 2020; https://nei.nih.gov/3droc). The competition requires participants to develop methods to improve organoid differentiation, scalability, and reproducibility in addition to present data that demonstrate their organoids could serve as

\footnotetext{
${ }^{1}$ National Eye Institute and ${ }^{2}$ National Center for Advancing Translational Sciences, National Institutes of Health, Bethesda, Maryland.
} 


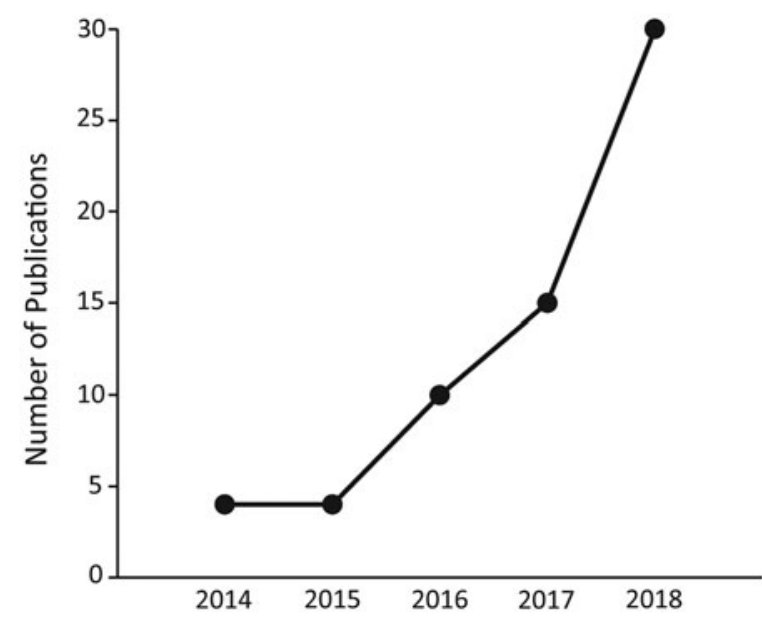

FIG. 1. Retinal organoid publications by year. The number of publications featuring or using retina organoids as an experimental tool each year from 2014 through September 2018 is shown. Publications were identified using "retinal organoid," "retinal sheet," "retinal neurosphere," "retinal spheroid," and "optic vesicle-like" as search terms in PubMed on Sept. 20, 2018. Unique publications were identified by PMID, and redundant search results were excluded from consideration.

novel platforms for disease modeling or drug screening. ${ }^{1}$ Although these are ambitious objectives, the vision research community is poised to rise to the challenge.

When retina organoids were first described, the morphological similarity to developing human retina was striking. ${ }^{2,3}$ In addition, the time course of expression of developmental markers and mature cell type markers closely mirrored the development of retinal tissue in vivo. ${ }^{4-7}$ This demonstrates that the vision research field now has a powerful tool to aid in the understanding of how retina tissue develops and matures.

However, producing organoids that reliably respond to light remains an elusive goal. For unknown reasons, retina organoids cultured by different groups gain light sensitivity only $\sim 15 \%$ of the time. ${ }^{6,8,9}$ A recent study optimized culture conditions to produce more mature light-sensitive tissue in a scalable manner by optimizing both cell seeding density and culture nutrient condition. ${ }^{6}$ When organoids were cultured individually in 96-well plates, these showed a marked increase in the percentage of organoids that exhibited light sensitivity by electrophysiological testing; retina organoids cultured in large groups in 6-well plates were sensitive to light $\sim 25 \%$ of the time, but $\sim 37 \%$ of organoids cultured individually were light sensitive. ${ }^{5}$ These increases in light sensitivity correlated with increased expression of MITF, a factor important in differentiation of the retinal pigmented epithelium (RPE); TFAP-2 $\alpha$, a factor closely linked to retinal development; and MATH5 and RBPMS, both of which are critical for retinal ganglion cell differentiation. ${ }^{10-14}$ Also associated with increased expression of these gene markers was an increased incidence of spontaneously generated RPE in individual organoid cultures. ${ }^{12}$ These results suggest efforts to increase scalability may help to create systems for large-scale drug screening and also improve differentiation protocols and reproducibility of retina organoids, possibly by improving the relative amount of nutrients available to organoids when they are individually cultured. ${ }^{12}$

In addition to optimizing cell seeding and nutrient conditions, other groups have used organoids to further dissect cell signaling and cellular processes critical to differentiation in health and disease. Cone cell health and cone opsin expression in the retina has long been understood to be heavily influenced by thyroid hormone, but its exact role has been unclear until recently. ${ }^{15} \mathrm{~A}$ group using retina organoids derived from human-induced pluripotent stem cells (iPSCs) recently determined thyroid hormone acts as a critical temporal switch in the differentiation of S-cones from M/Lcones. ${ }^{16}$ These retina organoids showed evidence of high concordance of gene expression when compared with human retina, suggesting these organoids could serve as valuable models for dissecting cell signaling events in development. ${ }^{16}$ Indeed, the findings in organoid cultures were found to be relevant to vision health in premature infants with low T3 and T4 hormone because these infants also exhibited deficits in color vision. ${ }^{16}$

Study focusing on retinitis pigmentosa 11 (RP11) patients with PRPF31 mutations demonstrated the utility of organoids in dissecting human disease. ${ }^{17}$ Mutations in ubiquitously expressed genes are expected to give rise to syndromic disease, but in some patients with inherited retinal degeneration, the disease phenotype is restricted solely to the eye. ${ }^{18}$ Such is the case in patients with mutations in PRPF31, a gene coding for a factor critical for spliceosome function. ${ }^{19}$ mRNA splicing requires proper spliceosome function in every living cell, but RP11 patients only suffer from retinal degeneration. ${ }^{18}$ RPE derived from RP11 patients show defects in RPE polarity and increased expression of angiogenic modulators $P E D F$ and $V E G F$, decreased transepithelial resistance, and impaired rod outer segment phagocytosis, and retina organoids derived from these cells show impaired cilia morphogenesis. ${ }^{17}$ These findings have not been previously associated with RP11 in human patients, and animal models for the disease have struggled to completely recapitulate key features of the disease. ${ }^{20,21}$ Perhaps more importantly, retina organoids derived from these patients allowed investigators to discover that the PRPF31 mutation affected a splice variant of the factor incorporated into spliceosomes in a retina-specific manner, finally providing the vision research community an explanation for the nonsyndromic phenotype exhibited by RP11 patients. ${ }^{17}$ Moreover, the PRPF31 mutation was amenable to gene repair and phenotypic rescue with CRISPR-Cas9 technology, suggesting organoids derived from patients are not only useful investigative tools for basic biology but also for preliminary testing of therapeutics. ${ }^{17}$ These studies provide critical proofs of concept for basic and clinical researchers and provide the foundation upon which future advances in organoid technologies can be made.

Recent advances in ocular organoids have gone beyond the neural retina and RPE. Two independent groups have pioneered organoid technology to develop miniature corneas that capture the morphology of the developing tissue. ${ }^{22,23}$ Current efforts coax iPSCs to an anterior neural commitment using Matrigel ${ }^{\circledR}$ scaffolds, retinoic acid exposure, and inhibition of Wnt and Notch signaling pathways, but the low efficiency of producing cornea organoids using this methodology suggests that much more refinement of the differentiation protocol is needed. ${ }^{22}$ Nevertheless, these cornea organoids recapitulate the structures of the human cornea in that they are semitransparent, produce corneal epithelium, have stroma characterized by organized collagen fibrils, contain a basement reminiscent of Descemet's membrane, 
and have a corneal endothelium. ${ }^{23}$ The identification of these cell types by morphological analysis is corroborated by gene expression analyses; corneal epithelium from these organoids expresses KRT3 and KRT14; corneal endothelium expresses COL8A1, F11R, and S1O0A4; and both epithelium and endothelium show low expression of the cornea precursor marker KRT12. ${ }^{23}$ In addition, stromal keratocyte markers $C D 34$ and KERA are readily detectable in the stroma of these organoids. ${ }^{23}$ These advances raise the intriguing possibility that cornea organoids could be used for the study of human diseases with unclear etiology, such as Fuchs' dystrophy or for the production of corneal cells that could be used for cell therapy approaches to corneal disease. .2,23 $^{2,2}$

Corneal tissue chip platforms that could be used for preclinical evaluations of therapeutic drugs are also in development. ${ }^{24}$ Dr. Dan Huh is working on an eye-on-a-chip that mimics blinking by incorporating a hydrogel "eyelid." ${ }^{25}$ As the human cornea has collagen-rich acellular layers that are also avascular, it is amenable to bioengineering approaches using cornea organoids and cornea tissue chips. This may impact the way ophthalmic drugs are developed and tested in the future. Collectively, these advances show that although ocular organoid and tissue chip technology are still evolving, the contributions they make to vision research are promising.

\section{Addressing Organoid Challenges with a Prize Competition}

The NEI 3-D ROC 2020 aims to catalyze innovation in retina organoids to surmount challenges facing organoid technology in general (Table 1). Many of the proposals received in the first phase of the competition sought to improve retinal organoid morphology and reproducibility using approaches that utilized microfluidic technology, advanced bioreactor design, and various bioengineering techniques (short descriptions of the ideation proposals can be found at https://nei.nih.gov/ideationwinner). In December 2018, a team led by Wei Liu, PhD, from the Albert Einstein College of Medicine was recognized in the first submission round of the challenge for their demonstration of cone-enriched retina organoids that had gene expression profiles similar to those found in the human macula.

Organoids derived from PSCs have been shown to recapitulate the genesis of various tissues with similar developmental timing. This means that human retina organoids need $>200$ days in culture before photoreceptors emerge and begin to have functional light responses. This long and in- tensive culturing time is one of the barriers to widespread adoption of retina organoids. Retina neurons derived from PSCs, even if light responsive, may be different than primary cells in other ways not yet appreciated. Such differences may be related to an epigenetic signature different than adult tissue. ${ }^{34}$

Inherent in a 3-D retina culture is the necessity to differentiate and maintain multiple cell types. The retina is composed of various cell types that require different localized signaling to form properly oriented laminated layers. Signaling pathways that control the development of various cell types are being elucidated, but integration of that knowledge to coax organoids to generate and maintain all the various cell types in the appropriate proportions and positions is lacking. Further complicating matters is the fact that some growth factors and cytokines that contribute to the maturation or differentiation of 1 cell type antagonizes the development and viability of another cell type. Improved universal media formulations, combined with nanotechnology that controls the release of growth factors and cytokines, may enable the complexity of the retina to be faithfully recapitulated. Another limitation of current organoids that may hinder differentiation and maturation is the lack of a vasculature to circulate nutrients and discard waste products. Although some groups are looking to add choroid to retinal organoids, others are looking to integrate microfluidics and scaffolds to allow for greater nutrient exchange.

Once the biology has been optimized as a drug development tool, drug companies would want the ability to screen compounds with high throughput. This means that new technologies with improved bioreactor design are critical for the mass production of organoids. ${ }^{30,31}$ For disease modeling things get more complicated when the etiology is unknown or the genetic and environmental contributions are complex or multifactorial. The lack of recapitulation of the microenvironment in terms of the extracellular matrix, immune cells, and vasculature, may limit the extent to which certain diseases can be modeled. Efforts to combine organoid and tissue chip technologies are ongoing and the merger of such technologies promises to reduce the time, cost, and use of animals required for new ocular therapies. ${ }^{35}$

\section{Catalyzing Tissue Chip Technology}

Ultimately, to be used for drug screening, both the organoid and tissue chip fields need to overcome the challenges of

Table 1. A Summary of the General Challenges to Organoid Development and Some Potential Solutions AND EXAMPLES TO ADDRESS THEM

\begin{tabular}{|c|c|c|}
\hline Organoid challenges & Potential solutions & Examples \\
\hline $\begin{array}{l}\text { Reduce maturation time and } \\
\text { improve differentiation }\end{array}$ & $\begin{array}{l}\text { Use of nanotechnology and direct } \\
\text { reprogramming; } ; 6 \text { use of scaffolds }{ }^{27} \\
\text { addition of glial cells }\end{array}$ & $\begin{array}{l}\text { Human pluripotent stem cell-derived } \\
\text { astrocytes enhance maturation of retinal } \\
\text { ganglion cells }\end{array}$ \\
\hline Minimize variability & $\begin{array}{l}\text { Use of scaffolds and bioreactors; } \\
\text { quantitative technologies } \\
\text { for analysis }\end{array}$ & $\begin{array}{l}\text { Bioreactors using mouse retina organoids; } \\
\text { brain organoids from miniaturized } \\
\text { multiwell spinning bioreactor (Spin } \Omega)^{31}\end{array}$ \\
\hline Increase tissue fidelity & Add microfluidics or vasculature & $\begin{array}{l}\text { Microfluidic bioprinting with human } \\
\text { umbilical vein endothelial cells }{ }^{32}\end{array}$ \\
\hline Enable scalability & Increased automation & $\begin{array}{l}\text { Generation of storable retinal organoids from } \\
\text { human-induced pluripotent stem cells } \\
\text { in xeno-free and feeder-free conditions }{ }^{33}\end{array}$ \\
\hline
\end{tabular}


reproducibility and validation. These barriers are not trivial and will require efforts from both biological and technical fields to address. The obstacles cut across a broad spectrum that range from cell sourcing and maturity issues to platform design using appropriate materials and fit-for-purpose cell scaffolds. ${ }^{36,37}$ As a starting point, the NIH has guidelines for cell authenticity (see "https://grants.nih.gov/grants/guide/ notice-files/NOT-OD-17-068.html" Authentication of Key Biological and/or Chemical Resources"') and for NIH-funded human stem cell research (https://stemcells.nih.gov/ policy/2009-guidelines.htm).

The National Center for Advancing Translational Sciences (NCATS) at the U.S. NIH has supported a number of efforts to catalyze the tissue-on-a-chip field and to spur the validation and commercialization efforts of the technology. Beginning in 2011, and alongside a sister program from the Defense Advanced Research Projects Agency (DARPA), NCATS has led multiple initiatives to develop and demonstrate the use of tissue-on-chip platforms, and has helped fund the creation of tissue chips for drug screening and toxicity testing, and more recently has funded researchers to create disease models on a chip for drug efficacy testing. The NIH and DARPA programs have facilitated progress from a number of angles. The DARPA program, focused on linking at least 10 organ systems into a "body on a chip," focused heavily on technical issues such as the integration of multiple microfluidic bioreactors that could maintain sterile cell culture and survival for the course of 1 month. The NIH programs focused more on the biological fidelity of the platforms, with critical factors such as the use of renewable cell sources, that is, primary cells and stem cells in lieu of cell lines, modeling of the in vivo cytoarchitecture, and exposure to appropriate biomechanical forces and environments being of high importance.

One way that NIH has helped address the challenges facing the organoid and tissue chip field is by involving end-user stakeholders at the outset, encouraging input and feedback from regulatory and pharmaceutical industry representatives. As a result, the U.S. Food and Drug Administration (FDA) has been an active participant in United States government-funded organoid and tissue chip development since the joint NIH-FDA initiative (https://common fund.nih.gov/regulatoryscience) on Advancing Regulatory Science program in 2010, which aimed to fund science that would accelerate the development and use of new tools, standards, and approaches to efficiently develop medical products and to more effectively evaluate medical product safety, efficacy, and quality. Among the awards made under this initiative was an early tissue chip project termed a "heart-lung micromachine" from the Wyss Institute. In addition, NCATS began an initiative in 2016 to create "Tissue Chip Testing Centers" (TCTCs), which were charged with onboarding multiple different platforms from laboratories around the United States and reproducing the findings in their independent laboratories, with both the FDA and the IQ Consortium as key advisors as to the most relevant assays and informative compounds that would validate each system. The IQ Consortium, which comprises membership from pharmaceutical and biotechnology industries, aims to advance innovative solutions to biomedical problems. They initially created an MPS working group that advised and consulted with the NCATS Tissue Chip Testing Centers. This working group has now been transformed into an Affiliate group, reflecting the growing interest from pharmaceutical industry tissue chip end-users. ${ }^{38}$
There are other institutes leveraging the progress of the NCATS tissue-on-a-chip efforts. To stimulate basic research to develop next-generation human cell-derived MPS that more closely recapitulates the cornea, lens, retina, RPE, and/or elements of the central visual pathway, the NEI has joined 4 other institutes to support a funding opportunity announcement for "Engineering Next-Generation Human Nervous System Microphysiological Systems" (https:// grants.nih.gov/grants/guide/pa-files/PAR-16-398.html).

$\mathrm{NEI}$ is interested in research that develops natural and/or synthetic substrates/scaffolds that promote the growth and differentiation of physiologically relevant tissue or utilizes microfluidic devices (eye-on-chip) derived from stem cells that model physiological functions of the visual system. In addition, ocular tissue platforms that model microenvironments or integrate complex functional features such as circuit structure, function, and connectivity in the visual system are also needed.

\section{Conclusions}

Retinal cell lines and explant cultures have been used in vision research to probe normal and diseased physiology. For 2dimensional cell culture, the interactions and influences of other supportive cell types has been tried with coculture methods, but the ability to recapitulate developmental cues at the appropriate time with the needed 3-D positional information is limited and approximate at best. Organoid and tissue chip technologies have already improved our understanding of developmental processes that are difficult to study in vivo. The ability to genetically dissect different signaling pathways that may arise in different cell types provides a system to explore questions with living human tissue that were previously not possible. The insights derived from comparing how normal eyes progress to disease conditions should enable the discovery of new drug targets and enhance in silico disease modeling. Once biotech and drug companies adopt and integrate these technologies, the pace of drug discovery and disease therapies for ocular conditions should accelerate further. By generating genetically diverse panels of ocular organoids or tissue chips, toxicology and efficacy profiles of ophthalmic drugs in different populations may be able to be predicted. Such panels would help herald in an era of precision medicine, where blinding diseases are first modeled in a dish or tissue chip and then screened for novel ocular therapies that can be translated back to patients.

\section{Author Disclosure Statement}

No competing financial interests exist.

\section{References}

1. Mazerik, J.M., Becker, S., and Sieving, P.A. 3-D retina organoids: building platforms for therapies of the future. Cell Med. 10:1-6, 2018.

2. Nakano, T., Ando, S., Takata, N., et al. Self-formation of optic cups and storable stratified neural retina from human ESCs. Cell Stem Cell. 10:771-785, 2012.

3. Eiraku, M., Takata, N., Ishibashi, H., et al. Self-organizing optic-cup morphogenesis in three-dimensional culture. Nature. 472:51-56, 2011.

4. Fligor, C.M., Langer, K.B., Sridhar, A., et al. Threedimensional retinal organoids facilitate the investigation of retinal ganglion cell development, organization and neurite outgrowth from human pluripotent stem cells. Sci. Rep. 8: 14520, 2018. 
5. Gagliardi, G., Ben M'Barek, K., Chaffiol, A., et al. Characterization and transplantation of CD73-positive photoreceptors isolated from human iPSC-derived retinal organoids. Stem Cell Rep. 11:665-680, 2018.

6. Hallam, D., Hilgen, G., Dorgau, B., et al. Human-induced pluripotent stem cells generate light responsive retinal organoids with variable and nutrient-dependent efficiency. Stem Cells. 36:1535-1551, 2018.

7. Wang, L., Hiler, D., Xu, B., et al. Retinal cell type DNA methylation and histone modifications predict reprogramming efficiency and retinogenesis in 3D organoid cultures. Cell Rep. 22:2601-2614, 2018.

8. Zhong, X., Gutierrez, C., Xue, T., et al. Generation of three-dimensional retinal tissue with functional photoreceptors from human iPSCs. Nat. Commun. 5:4047, 2014.

9. Sridhar, A., Ohlemacher, S.K., Langer, K.B., and Meyer, J.S. Robust differentiation of mRNA-reprogrammed human induced pluripotent stem cells toward a retinal lineage. Stem Cells Transl. Med. 5:417-426, 2016.

10. Adijanto, J., Castorino, J.J., Wang, Z.X., et al. Microphthalmia-associated transcription factor (MITF) promotes differentiation of human retinal pigment epithelium (RPE) by regulating microRNAs-204/211 expression. J. Biol. Chem. 287:20491-20503, 2012.

11. Capowski, E.E., Simonett, J.M., Clark, E.M., et al. Loss of MITF expression during human embryonic stem cell differentiation disrupts retinal pigment epithelium development and optic vesicle cell proliferation. Hum. Mol. Genet. 23:6332-6344, 2014.

12. Jain, S., Glubrecht, D.D., Germain, D.R., Moser, M., and Godbout, R. AP-2epsilon expression in developing retina: contributing to the molecular diversity of amacrine cells. Sci. Rep. 8:3386, 2018.

13. Tanaka, T., Yokoi, T., Tamalu, F., et al. Generation of retinal ganglion cells with functional axons from human induced pluripotent stem cells. Sci. Rep. 5:8344, 2015.

14. Kwong, J.M., Caprioli, J., and Piri, N. RNA binding protein with multiple splicing: a new marker for retinal ganglion cells. Invest. Ophthalmol. Vis. Sci. 51:1052-1058, 2010.

15. Ma,H., andDing, X.Q. Thyroid hormone signaling and cone photoreceptor viability. Adv. Exp. Med. Biol. 854:613-618, 2016.

16. Eldred, K.C., Hadyniak, S.E., Hussey, K.A., et al. Thyroid hormone signaling specifies cone subtypes in human retinal organoids. Science. 362:eaau6348, 2018.

17. Buskin, A., Zhu, L., Chichagova, V., et al. Disrupted alternative splicing for genes implicated in splicing and ciliogenesis causes PRPF31 retinitis pigmentosa. Nat. Commun. 9:4234, 2018.

18. Baehr, W., and Chen, C.K. RP11 and RP13: unexpected gene loci. Trends Mol. Med. 7:484-486, 2001.

19. Schaffert, N., Hossbach, M., Heintzmann, R., Achsel, T., and Luhrmann, R. RNAi knockdown of hPrp31 leads to an accumulation of U4/U6 di-snRNPs in Cajal bodies. EMBO J. 23:3000-3009, 2004.

20. Bujakowska, K., Maubaret, C., Chakarova, C.F., et al. Study of gene-targeted mouse models of splicing factor gene Prpf31 implicated in human autosomal dominant retinitis pigmentosa (RP). Invest. Ophthalmol. Vis. Sci. 50:5927-5933, 2009.

21. Graziotto, J.J., Farkas, M.H., Bujakowska, K., et al. Three gene-targeted mouse models of RNA splicing factor RP show late-onset RPE and retinal degeneration. Invest. Ophthalmol. Vis. Sci. 52:190-198, 2011.

22. Foster, J.W., Wahlin, K., Adams, S.M., et al. Cornea organoids from human induced pluripotent stem cells. Sci. Rep. 7:41286, 2017.
23. Susaimanickam, P.J., Maddileti, S., Pulimamidi, V.K., et al. Generating minicorneal organoids from human induced pluripotent stem cells. Development. 144:2338-2351, 2017.

24. Bennet, D., Estlack, Z., Reid, T., and Kim, J. A microengineered human corneal epithelium-on-a-chip for eye drops mass transport evaluation. Lab. Chip. 18:1539-1551, 2018.

25. Seo, J., Byun, W.Y., and Frank, A. Human blinking eye-ona-chip. Invest. Ophthalmol. Vis. Sci. 57:3872, 2016.

26. Fang, L., El Wazan, L., Tan, C., et al. Potentials of cellular reprogramming as a novel strategy for neuroregeneration. Front. Cell Neurosci. 12:460, 2018.

27. Singh, D., Wang, S.B., Xia, T., et al. A biodegradable scaffold enhances differentiation of embryonic stem cells into a thick sheet of retinal cells. Biomaterials. 154:158-168, 2018.

28. VanderWall, K.B., Vij, R., Ohlemacher, S.K., et al. Astrocytes regulate the development and maturation of retinal ganglion cells derived from human pluripotent stem cells. Stem Cell Rep. 12:201-212, 2019.

29. Vergara, M.N., Flores-Bellver, M., Aparicio-Domingo, S., et al. Three-dimensional automated reporter quantification (3D-ARQ) technology enables quantitative screening in retinal organoids. Development. 144:3698-3705, 2017.

30. DiStefano, T., Chen, H.Y., Panebianco, C., et al. Accelerated and improved differentiation of retinal organoids from pluripotent stem cells in rotating-wall vessel bioreactors. Stem Cell Rep. 10:300-313, 2018.

31. Qian, X., Jacob, F., Song, M.M., et al. Generation of human brain region-specific organoids using a miniaturized spinning bioreactor. Nat. Protoc. 13:565-580, 2018.

32. Chen, L.J., Ito, S., Kai, H., et al. Microfluidic co-cultures of retinal pigment epithelial cells and vascular endothelial cells to investigate choroidal angiogenesis. Sci. Rep. 7:3538, 2017.

33. Reichman, S., Slembrouck, A., Gagliardi, G., et al. Generation of storable retinal organoids and retinal pigmented epithelium from adherent human iPS cells in xeno-free and feeder-free conditions. Stem Cells. 35:1176-1188, 2017.

34. Berry, B.J., Smith, A.S.T., Young, J.E., and Mack, D.L. Advances and current challenges associated with the use of human induced pluripotent stem cells in modeling neurodegenerative disease. Cells Tissues Organs. 1-19, 2018.

35. Takebe, T., Zhang, B., and Radisic, M. Synergistic engineering: organoids meet organs-on-a-chip. Cell Stem Cell. 21:297-300, 2017.

36. Liu, Y., Gill, E., and Shery Huang, Y.Y. Microfluidic onchip biomimicry for 3D cell culture: a fit-for-purpose investigation from the end user standpoint. Future Sci. O.A. 3:FSO173, 2017.

37. Low, L.A., and Tagle, D.A. Tissue chips-innovative tools for drug development and disease modeling. Lab. Chip. 17: 3026-3036, 2017.

38. Ewart, L., Fabre, K., Chakilam, A., et al. Navigating tissue chips from development to dissemination: a pharmaceutical industry perspective. Exp. Biol. Med. (Maywood). 242: 1579-1585, 2017.

Received: December 11, 2018 Accepted: March 20, 2019 Address correspondence to: Dr. Steven M. Becker National Eye Institute

National Institutes of Health 31 Center Drive, Rm $6 \mathrm{AO} 3$ Bethesda, MD 20892

E-mail: steven.becker@nih.gov 